\title{
Generalized Duffy transformation for integrating vertex singularities
}

\author{
S. E. Mousavi · N. Sukumar
}

Received: 15 July 2009 / Accepted: 24 September 2009 / Published online: 16 October 2009

(C) The Author(s) 2009. This article is published with open access at Springerlink.com

\begin{abstract}
For an integrand with a $1 / r$ vertex singularity, the Duffy transformation from a triangle (pyramid) to a square (cube) provides an accurate and efficient technique to evaluate the integral. In this paper, we generalize the Duffy transformation to power singularities of the form $p(\mathbf{x}) / r^{\alpha}$, where $p$ is a trivariate polynomial and $\alpha>0$ is the strength of the singularity. We use the map $(u, v, w) \rightarrow(x, y, z): x=u^{\beta}$, $y=x v, z=x w$, and judiciously choose $\beta$ to accurately estimate the integral. For $\alpha=1$, the Duffy transformation ( $\beta=1$ ) is optimal, whereas if $\alpha \neq 1$, we show that there are other values of $\beta$ that prove to be substantially better. Numerical tests in two and three dimensions are presented that reveal the improved accuracy of the new transformation. Higher-order partition of unity finite element solutions for the Laplace equation with a derivative singularity at a re-entrant corner are presented to demonstrate the benefits of using the generalized Duffy transformation.
\end{abstract}

Keywords Weakly singular integrand - Numerical quadrature - Partition of unity enrichment - FEM - BEM

\section{Introduction}

Elliptic boundary-value problems can admit solutions $u \sim r^{\lambda}$ $(0<\lambda<1)$ with derivative singularities-Laplace equation in a domain with a re-entrant corner or when there is an abrupt change in the boundary condition (Dirichlet to Neumann) [1,2]; problems in elasticity such as analysis of

National Science Foundation, CMMI-0626481, DMS-0811025.

S. E. Mousavi · N. Sukumar $(\bowtie)$

Department of Civil and Environmental Engineering,

University of California, Davis, CA 95616, USA

e-mail: nsukumar@ucdavis.edu plates with sharp notches and cracks [3], wedge-shaped bimaterials $[4,5]$, crack impinging a bimaterial interface [6], and bimaterial interfacial cracks [7] are well-known examples. In the numerical treatment of such problems with the boundary element and enriched finite element methods, the numerical integration of weakly singular integrands of $\mathcal{O}\left(1 / r^{\alpha}\right)$ arises where $0<\alpha<n$ in $\mathbb{R}^{n}$. The development of meshfree [8] and partition of unity finite element methods [9] for modeling singularities-cracks in isotropic media $(\lambda=1 / 2)$ [10-13], strong and weak singularities for a crack perpendicular to a bimaterial interface [14], complete sliding contact [15], HRR crack-tip fields with $\lambda=1 /(n+1)(n$ is the hardening exponent) [16], hydraulic fracture $(\lambda=1 / 2,2 / 3)$ $[17,18]$, and parametric enrichment for singular problems ( $\lambda$ is itself a parameter, which is obtained through optimization in the solution procedure) [19]—underscores the need to develop accurate numerical integration schemes to compute weak form integrals, which provides the impetus for pursuing this contribution.

The use of standard integration techniques such as GaussLegendre quadrature rules to evaluate singular integrands has its limitations, both, from the accuracy and cost perspectives. Extrapolation techniques [20-23] construct more accurate integration formulae based on asymptotic error expansions of standard quadratures, whereas in adaptive subdivision scheme $[24,25]$ the integration domain is subdivided into uniform/nonuniform subdomains and well-known integration rules are used over the subdomains. Klees [26] shows that for weakly singular integrands, extrapolation and adaptive subdivision techniques behave poorly in terms of both accuracy and efficiency.

Variable transformation methods (also referred as cancelation schemes) to numerically integrate weakly singular integrands are well-established in the literature [27-33]. The main idea in this approach is to map the physical domain to a 


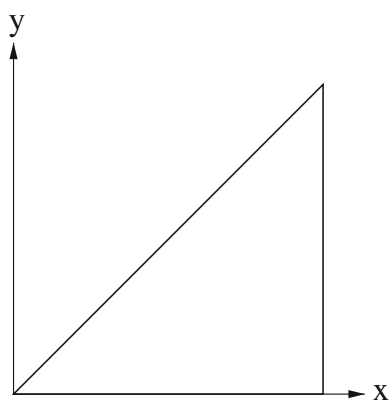

(a) $0 \leq x \leq 1,0 \leq y \leq x$

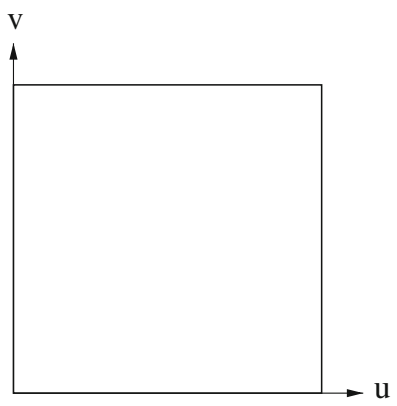

(b) $0 \leq u, v \leq 1$
Fig. 1 Duffy transformation from (a) the standard triangle to (b) the unit square

parent domain so that the singularity is removed through the introduction of the Jacobian. Among the transformation techniques, the so-called Duffy transformation has found wide appeal. The Duffy transformation [28] from a triangle (pyramid) to a square (cube) is: $(u, v, w) \rightarrow(x, y, z): x=u$, $y=x v=u v, z=x w=u w$, which eliminates singularities of the type $1 / r$ (see Fig. 1). The mapped kernel over the square (cube) is smooth enough and can be integrated within a desired accuracy using a tensor-product Gauss quadrature rule. When the singularity falls inside an element, then the element can be divided into triangles in $\mathbb{R}^{2}$ (pyramids in $\mathbb{R}^{3}$ ) with the singularity lying at a vertex of the subdivisions and the transformation can be applied to each subdomain separately.

As noted by Monegato and Scuderi [34], though attributed to Duffy, the above transformation in two dimensions was proposed earlier by Fairweather et al. [35] to numerically integrate a $1 / r$ vertex singularity over a triangle. For the twodimensional integral considered by Duffy [28], the integrand had a $u^{-1 / 2}$ term and therefore Gauss-Jacobi quadrature rule was used in the $u$-direction, and Gauss quadrature in the $v$-direction. However, in most boundary element and finite element applications, it is more convenient to adopt standard Gauss quadrature in all directions. The Duffy transformation has been used for the integration of stiffness matrix entries with singular finite element shape function derivatives [36-40]. The Duffy transformation has also been applied within boundary element and finite element methods: applications in Stokes flow [41], wave scattering and Helmholtz equation [42-44], and quantum mechanical density-functional calculations [45-48] to name a few.

Many recent studies have tackled the issue of numerical integration of singularities within partition of unity finite element methods [49-53]. Laborde et al. [49] triangulate the element with the singularity inside it so that the source point lies at a vertex of a triangle and then the Duffy transformation (though not mentioned in Ref. [49]) is applied to integrate singular functions over the triangle. This technique proves to be more accurate and has a better convergence rate than standard Gauss quadrature.

Other types of mappings have also been employed for different classes of functions and singularities. For example, Nagarajan and Mukherjee [32] use a polar mapping $(\rho, \theta) \rightarrow(x, y): x=\rho \cos ^{2} \theta, y=\rho \sin ^{2} \theta$, which transforms a master triangular element to a square element and as a result the $1 / r$ singularity is removed. Park et al. [53] generalize this transformation to three-dimensional tetrahedral elements and use it to integrate singular enrichment bases for crack problems within the generalized finite element method. The main advantage of this mapping is in the integration of terms such as $f(\mathbf{x}) / r$, where $f$ is a homogeneous function. This permits integration with respect to $r$ to be carried out algebraically and the multiple integral in two (three) dimensions is reduced to a line (surface) integral, which permits machine-precision accuracy to be realized [32]. If $f$ is nonhomogeneous, the accuracy and efficiency of this technique is significantly reduced [53]. Béchet et al. [50] present a series of transformations in two dimensions, which eventually cancels $1 / r^{\alpha}$ singularities, but as they point out, their mapping does not readily extend to three dimensional domains.

Even though the Duffy transformation works very well for $1 / r$ singularity, it is not as efficient for $1 / r^{\alpha}$ when $\alpha \neq 1$. For partition of unity finite element applications with corners or cracks, the integrand in the stiffness matrix may consist of terms with singularities $\alpha<1$ and $\alpha \geq 1$. The aim of this paper is to present a generalization of the Duffy transformation that can provide improved accuracy for integrating vertex singularities within two- and three-dimensional domains. In Sect.2, we introduce the generalized transformation, and in Sect. 3, we compare its performance with the Duffy transformation. Numerical studies on the accuracy and convergence rate of the new transformation are presented in Sects. 3.13.3. In Sect. 3.4, application to the Laplace equation with a re-entrant corner is presented, where the rate of convergence in strain energy is studied for finite element (FE) and higher-order partition of unity finite element (PUFE) methods. We close with the main findings and a few final remarks in Sect. 4.

\section{Formulation}

A generalization of the Duffy transformation is proposed: $(u, v, w) \rightarrow(x, y, z): x=u^{\beta}, y=x v^{\gamma}=u^{\beta} v^{\gamma}, z=$ $x w^{\zeta}=u^{\beta} w^{\zeta}$, where $\beta, \gamma$ and $\zeta$ are selected so that the transformed kernel is as smooth as possible and can be integrated with the fewest number of evaluation points. This transformation maps the standard pyramid with vertices at $(0,0,0)$, $(1,0,0),(1,1,0),(1,0,1)$ and $(1,1,1)$ to a unit cube [28]. Equation (1) shows the calculation of the integral in three 
dimensions after the transformation:

$$
\begin{aligned}
\mathcal{I} & =\int_{0}^{1} d x \int_{0}^{x} d y \int_{0}^{x} d z \frac{f(x, y, z)}{\left(x^{2}+y^{2}+z^{2}\right)^{\alpha / 2}} \\
& =\int_{0}^{1} \int_{0}^{1} \int_{0}^{1} \frac{f\left(u^{\beta}, u^{\beta} v^{\gamma}, u^{\beta} w^{\zeta}\right)}{\left[u^{2 \beta}\left(1+v^{2 \gamma}+w^{2 \zeta}\right)\right]^{\alpha / 2}} \mathcal{J} d u d v d w,
\end{aligned}
$$

where $\mathcal{J}$ is the Jacobian of the transformation:

$$
\mathcal{J}=\beta \gamma \zeta u^{3 \beta-1} v^{\gamma-1} w^{\zeta-1}
$$

From the expression for the Jacobian and the denominator in (1a), it is evident that the choice $\gamma=1$ and $\zeta=1$ provides the lowest exponents of the variables $v$ and $w$ in the transformed space. On using $\gamma=\zeta=1$ (1) reduces to

$\mathcal{I}=\int_{0}^{1} \int_{0}^{1} \int_{0}^{1} \frac{f\left(u^{\beta}, u^{\beta} v, u^{\beta} w\right)}{\left(1+v^{2}+w^{2}\right)^{\alpha / 2}} \beta u^{3 \beta-1-\alpha \beta} d u d v d w$,

where $\beta$ is now selected so that both $f\left(u^{\beta}, u^{\beta} v, u^{\beta} w\right)$ and $u^{3 \beta-1-\alpha \beta}$ have the simplest possible forms that can be easily integrated. Since in boundary element and finite element applications, polynomial bases are used and the integration of polynomials is required, we pick $\beta$ so that $f$ remains a polynomial in the transformed space. This requirement will marginally increase the polynomial order of $f$, and therefore require a slight increase in the order of the quadrature rule to exactly integrate the polynomial in the $u$-direction. However, a fractional exponent in the term $u^{3 \beta-1-\alpha \beta}$ needs a much higher-order quadrature rule to capture the non-linear behavior of the integrand. Therefore, we select the minimum $\beta \in \mathbb{Z}^{+}$so that the exponent $3 \beta-1-\alpha \beta$ is a positive integer. For instance, for a $1 / \sqrt{r}(\alpha=1 / 2)$ singularity, $\beta=2$ is chosen, which results in the term $u^{4}$, whereas in the Duffy transformation $(\beta=1)$, the term $u^{3 / 2}$ is present which leads to loss in accuracy. Similar arguments apply in the twodimensional case (Fig. 1); the generalized transformation in two dimensions is provided in Appendix A. We point out that for singularities $\alpha>2$ in three dimensions $(\alpha>1$ in two dimensions), the Duffy transformation does not remove the singularity since the exponent of $u$ in the transformed kernel is negative.

\section{Numerical examples}

We present numerical tests to affirm the improvements in accuracy that are realized by the generalized transformation vis-à-vis the Duffy transformation. The performance of the proposed transformation in the numerical integration of a vertex singularity in a square and a cube are presented. Finally, the Laplace problem on an $L$-shaped domain with a corner singularity is considered and the accuracy and rate of convergence of higher-order FE and PUFE solutions are studied.

\subsection{Vertex singularity in two dimensions}

To validate the criterion for the selection of $\beta$, we apply the Duffy transformation and the generalized transformation for the integration of functions $p(x, y) / r^{\alpha}$, where $p(x, y) \in$ $P_{d}(x, y)$ are bivariate polynomials up to degree $d$ with respect to $x$ and $y$ and $r$ is the distance from the origin. The integration is carried out over a unit square after dividing it into two triangles (Fig. 2a, b). Each triangle is mapped to the standard triangle of Fig. 1 through a shift of the coordinates and an affine mapping (see Appendix A). Different values of the singularity-exponent $\alpha$ are considered. We choose $\alpha=\{1,1 / 2,1 / 3,2 / 3,4 / 3\}$, which appear in various applications: $\alpha=1 / 2,1$ for cracks in isotropic media [10], $\alpha=$ $1 / 2,2 / 3$ in hydraulic fracture [18], and $\alpha=1 / 3,2 / 3,4 / 3$ in the solution of Laplace equation in an $L$-shaped domain with a re-entrant corner (see Sect. 3.4). For each $\alpha$, different choices of $\beta$ are tried. For example, Fig. 2c shows the convergence curves for $1 / r$ singularity with $\beta$ varying from 1 to 5 . Also, tensor-product quadrature rules over the triangulated domain and the unit square are tested (indicated as tensor 1 and tensor 2, respectively). The reported relative errors are the norm of the relative error in the integration of $p(x, y) / r^{\alpha}$, where $p$ includes all bivariate polynomials up to order three (ten functions in two dimensions). Figure $2 \mathrm{~d}$ to $g$ show similar results for other values of $\alpha$. The reference values are calculated using very high-order quadrature rules so that sufficient number of digits are converged. For some of the values of $\alpha$, e.g., $\alpha=1$ and $\alpha=1 / 2$, the reference integral is evaluated using symbolic packages such as Maple ${ }^{\mathrm{TM}}$ and MATLAB ${ }^{\mathrm{TM}}$ either exactly or with very high accuracy. For all values of $\alpha$ and $\beta$, the maximum error in the integration as $n s p$ increases corresponds to $\int\left(1 / r^{\alpha}\right) d x d y$, i.e., for a constant in the numerator of the integrand. This is due to the fact that bivariate polynomials $x^{i} y^{j}$ for $i+j>0$ have a radial dependence and therefore $\int\left(x^{i} y^{j} / r^{\alpha}\right) d x d y$ has a milder radial singularity than $\int\left(1 / r^{\alpha}\right) d x d y$. In accordance with our expectations, we note from Fig. 2 that the choice $\beta=\{1,2,3,3,3\}$ gives us the most accurate results for the singularities $\alpha=\{1,1 / 2,1 / 3,2 / 3,4 / 3\}$, respectively. Moreover, it is evident from the plots that if $\alpha \neq 1$, then the optimal $\beta$ delivers markedly better accuracy than the Duffy transformation $(\beta=1)$. Figure $2 \mathrm{c}$ to g also reveal that GaussLegendre quadrature has a very low convergence rate and the errors are $\mathcal{O}\left(10^{-5}\right)$ for 500 integration points. A MATLAB code that performs the generalized Duffy transformation is provided in Appendix B.

To further illustrate the performance of the generalized transformation, we apply it to the integration of functions with singularities $\alpha=p / q$ when $p$ and $q$ are moderately 


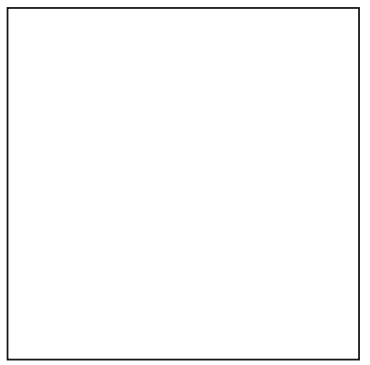

(a)

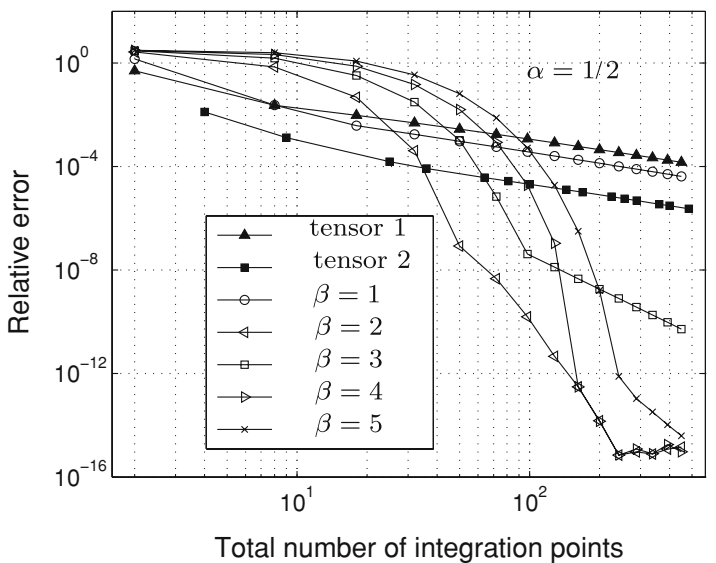

(d)

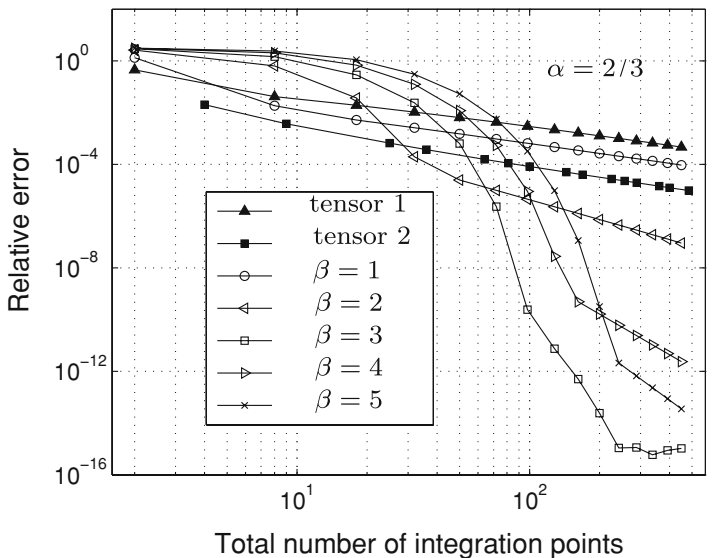

(f)

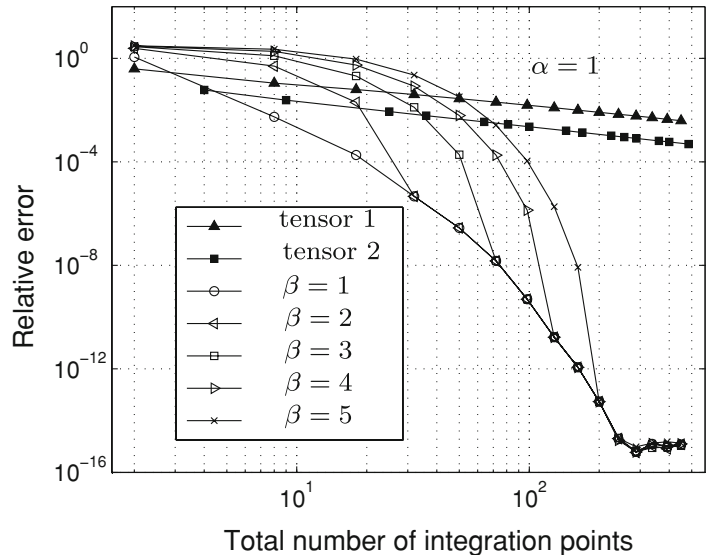

(c)

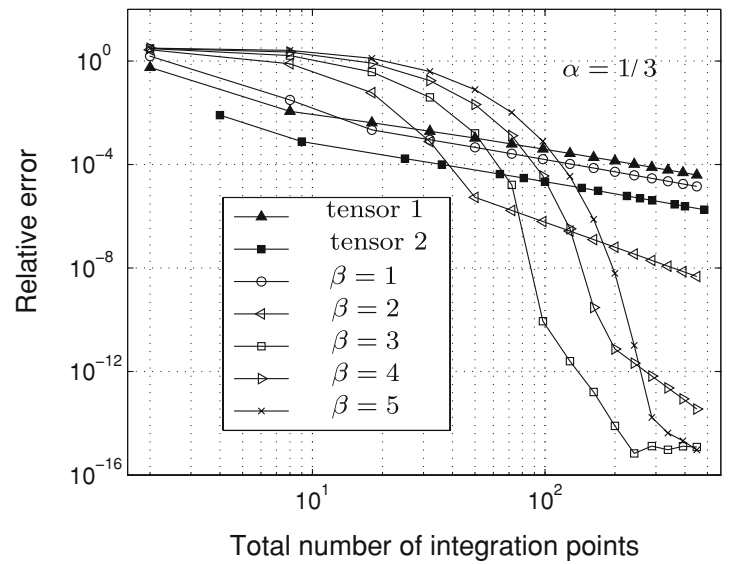

(e)

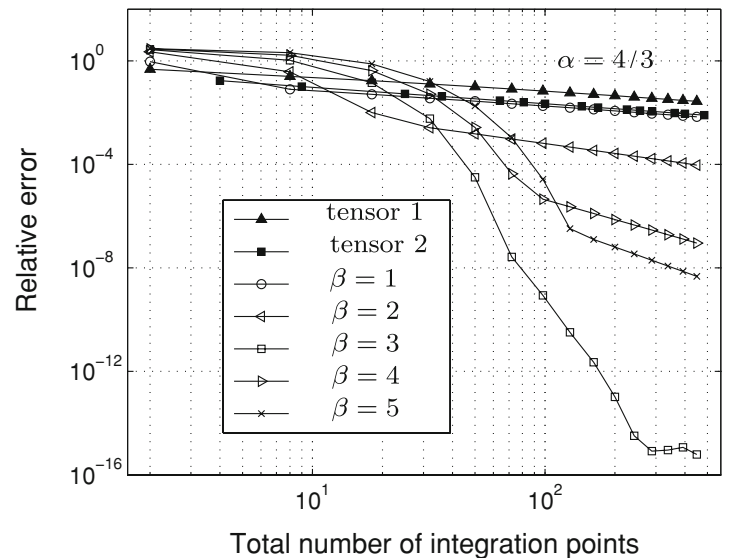

(g)

Fig. 2 Convergence curves for integration of $p(\mathbf{x}) / r^{\alpha}$ over a unit square. (a) Unit square and its (b) triangulation. Relative errors for (c) $\alpha=1$, (d) $\alpha=1 / 2,(\mathbf{e}) \alpha=1 / 3$, (f) $\alpha=2 / 3$, and (g) $\alpha=4 / 3$

large positive integers with no common divisor. Such values of $\alpha$ arise in re-entrant corner problems in orthotropic media [54], and in partition-of-unity enriched finite element methods with parametric enrichment functions [19]. The rationale provided in Sect. 2 for selecting $\beta$ would lead to the choice $\beta=q$. It should be noted that increasing $\beta$ has a dual effect: (1) Improves the accuracy due to the larger exponent of $u$ in the transformed kernel (the transformed domain of integration is the unit square and therefore $0 \leq u \leq 1$ ); and (2) More integration points are needed in the unit square 


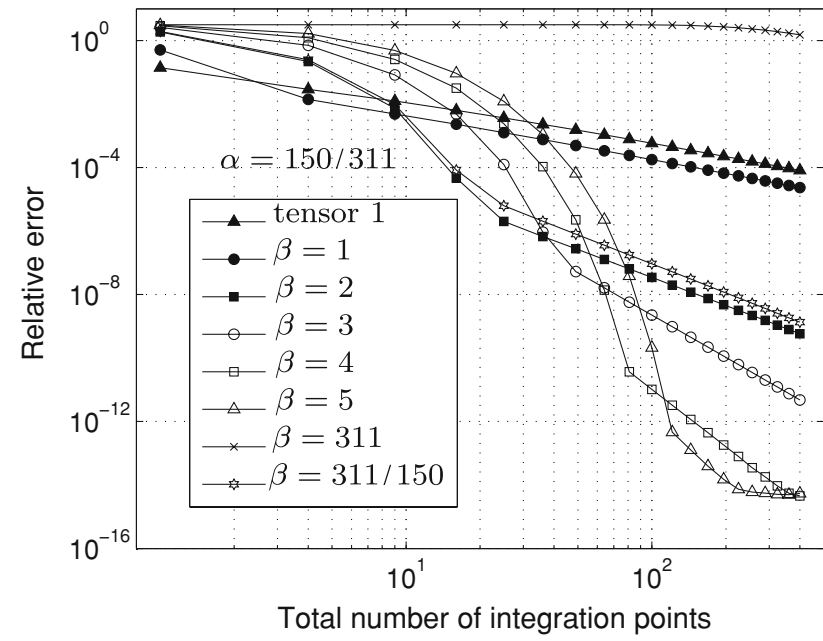

Fig. 3 Convergence curves for integration of $p(\mathbf{x}) / r^{\alpha}$ over the triangle with vertices $(1,1) ;(3,2)$ and $(1.5,2.3)$. Singularity is at $(1,1)$ and $\alpha=150 / 311$

due to the increase in the polynomial order of the transformed kernel. For such $\alpha$, numerical tests can guide the choice for $\beta$. As an example, for $\alpha=150 / 311$, we consider $\beta=\{1,2,3,4,5,311,311 / 150\}$. Figure 3 shows that an accuracy of $\mathcal{O}\left(10^{-8}\right)$ is realized on using the generalized Duffy transformation with $\beta=4$ and a $8 \times 8$ tensor product over the unit square.

\subsection{Vertex singularity in three dimensions}

We examine the three-dimensional case by integrating functions of the form $p(x, y, z) / r^{\alpha}$ over a unit cube that have a singularity at the origin. The function $p$ consists of trivariate polynomials up to degree three with respect to $x, y$, and $z$ (twenty functions in three dimensions). The unit cube is divided into three pyramids with planar bases, and each of these pyramids is mapped to the standard one and then the generalized transformation is applied. The unit cube is shown in Fig. 4a and one of its partitions (standard pyramid) is depicted in Fig. 4b. Similar to the the two-dimensional case, different singularities $\alpha=\{1,1 / 2,1 / 3,2 / 3,4 / 3\}$ are tested with $\beta$ varying from 1 to 5 . The results are plotted in Fig. $4 \mathrm{c}$ to g, and once again, the best choices of $\beta$ are identical to those obtained in the two-dimensional case. The optimal $\beta$ outperforms all other values of $\beta$ and is able to reduce the relative error to close to machine precision in all cases. For a relative error of $10^{-8}$, the optimal $\beta$ requires about 1,000 integration points; the tensor-product Gauss rule can at best deliver an accuracy of $10^{-7}$ with 10,000 integration points. Similar to the two-dimensional case, the constant term in the numerator of the integrand dominates the error in the numerical integration depicted in Fig. 4.

\subsection{Optimization}

Careful observation of the behavior of our generalized transformation shows that by increasing the number of integration points in each direction, the rate of convergence has a sudden decrease at a point and after that it converges with a much lower rate (for example, see the curve for $\beta=2$ in Fig. 2d). A similar behavior is seen in almost all the convergence curves. Further inspection reveals that the kernel after transformation remains a polynomial with respect to $u$, but is an irrational function with respect to $v$ and $w$. Hence, one can determine the number of Gauss points in the $u$-direction to obtain exact integration with respect to $u$, after which increasing the number of integration points in the $u$-direction does not have any effect on the accuracy. Based on this finding, we propose to use the minimum number of Gauss points in the $u$-direction to exactly evaluate the integral with respect to $u$, and a higher-order quadrature rule in the other directions. This idea is made more precise through the following example.

Consider an integrand of the form $p(x, y) / r^{\alpha}$, with $p(x, y)$ consisting of polynomials up to order $d$. As indicated in Appendix A, the kernel $K(u, v)$ after the transformation in two dimensions is

$K(u, v)=\frac{p\left(u^{\beta}, u^{\beta} v\right)}{\left(1+v^{2}\right)^{\alpha / 2}} \beta u^{2 \beta-1-\alpha \beta}$.

Collecting like-terms reveals that the highest exponent of $u$ is $2 \beta-1-\alpha \beta+d \beta$. Thus, it is sufficient to use $n s p_{u}=(2 \beta-$ $\alpha \beta+d \beta) / 2$ Gauss points in the $u$-direction to exactly evaluate the integral with respect to $u$. To obtain higher precision one only needs to increase the number of evaluation points in the other directions. Figure $5 \mathrm{a}$ and $\mathrm{b}$ show the convergence curves for $\alpha=1 / 2$ over the unit square and $\alpha=4 / 3$ over the unit cube, respectively. When $\beta=3$ (optimized) is used, the high convergence rate is maintained and in comparison to $\beta=3$ (without optimization), fewer number of integration points are needed to attain higher accuracies.

\section{4 $L$-shaped domain with a re-entrant corner}

The $L$-shaped domain with a corner singularity is a wellknown benchmark problem, which has been considered in previous studies $[12,55,56]$. The boundary-value problem is posed as:

$-\nabla^{2} u=0$ in $\Omega$,

$u=0$ on $\Gamma_{D}$

$\frac{\partial u}{\partial n}=g$ on $\Gamma_{N}$,

where $\Omega$ is the $L$-shaped domain shown in Fig. 6 . 


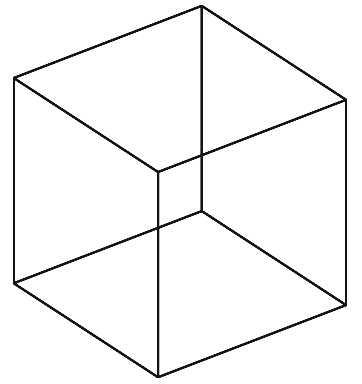

(a)

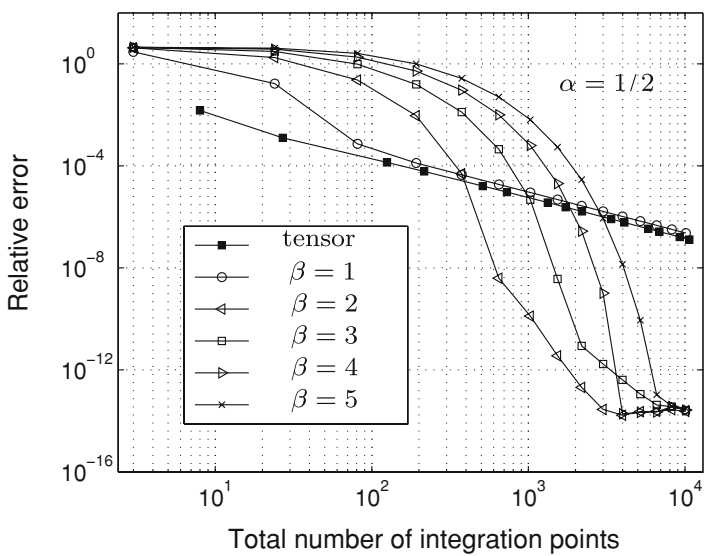

(d)

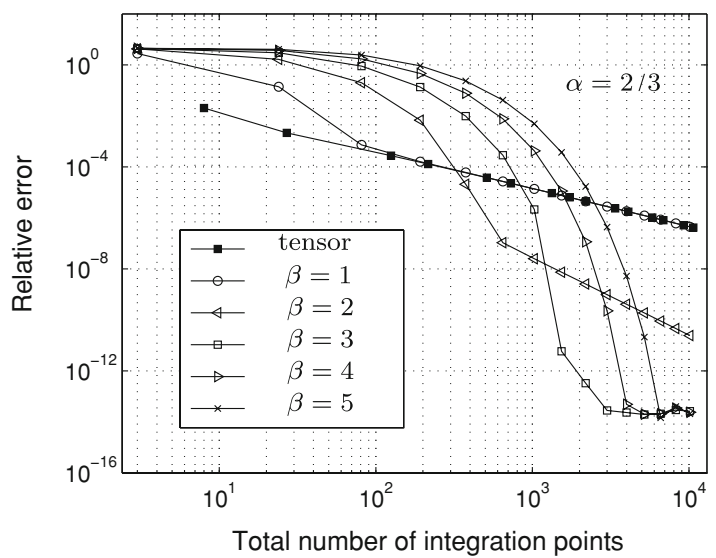

(f)

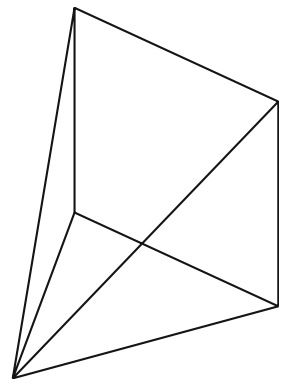

(b)

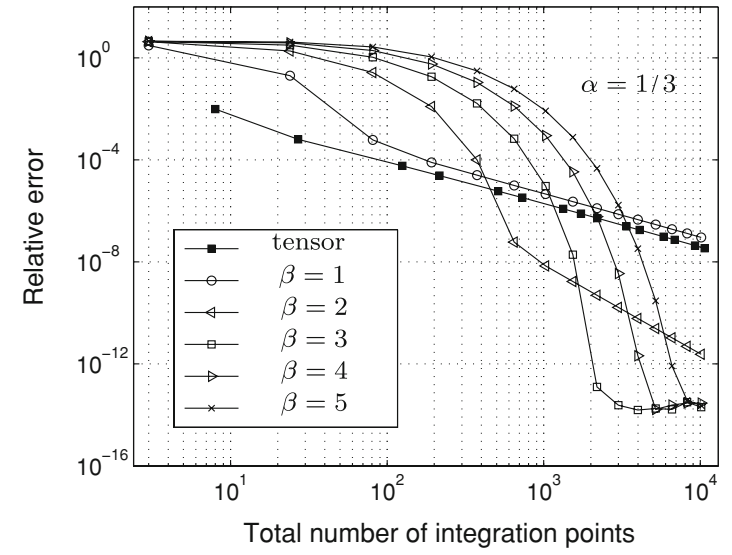

(e)

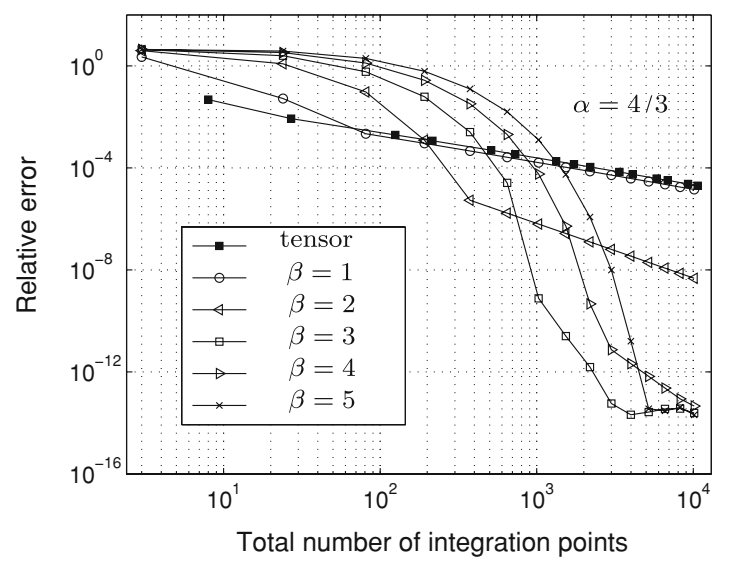

(g)

(c)

Fig. 4 Convergence curves for integration of $p(\mathbf{x}) / r^{\alpha}$ over a unit cube. (a) Unit cube and one of its partitions, (b) the standard pyramid. Relative errors for (c) $\alpha=1$, (d) $\alpha=1 / 2$, (e) $\alpha=1 / 3$, (f) $\alpha=2 / 3$, and (g) $\alpha=4 / 3$

Following Strouboulis et al. [12], we choose boundary conditions that are consistent with the exact solution:

$u=r^{1 / 3} \sin \frac{\theta}{3}, \quad \nabla u=\frac{r^{-2 / 3}}{3}\left[-\sin \frac{2 \theta}{3} \mathbf{i}+\cos \frac{2 \theta}{3} \mathbf{j}\right]$

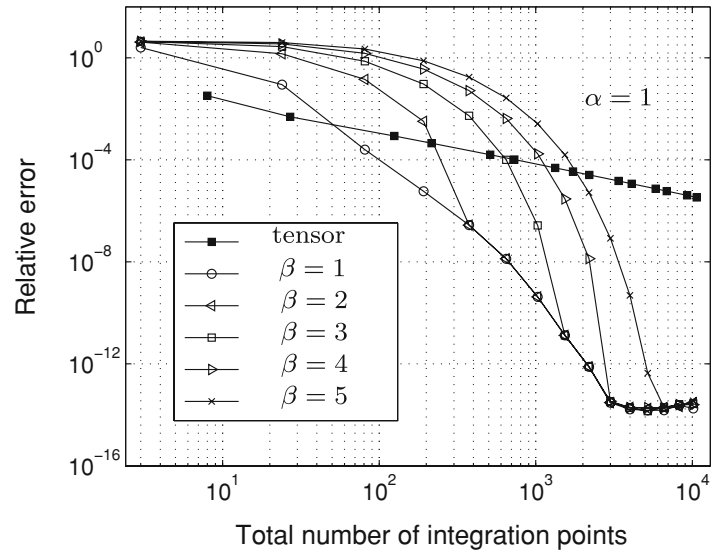




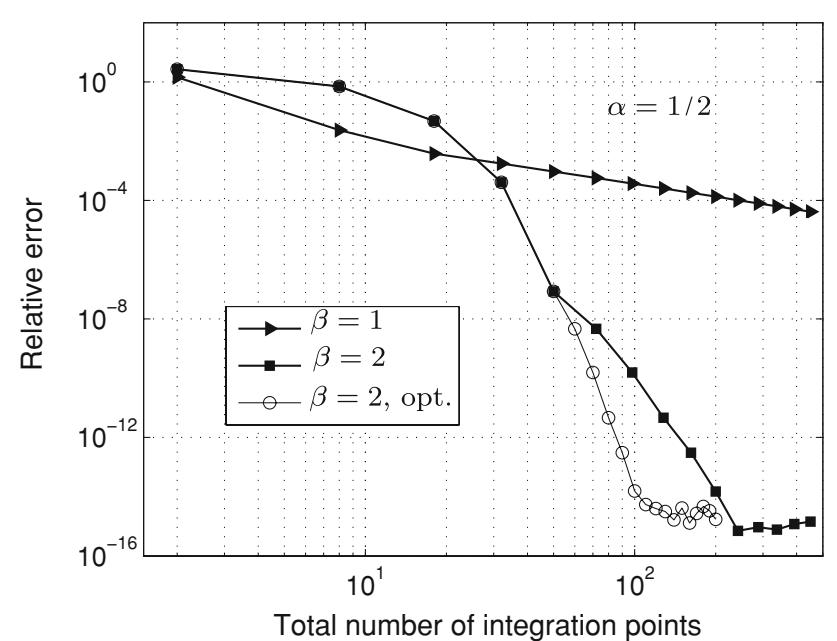

(a)

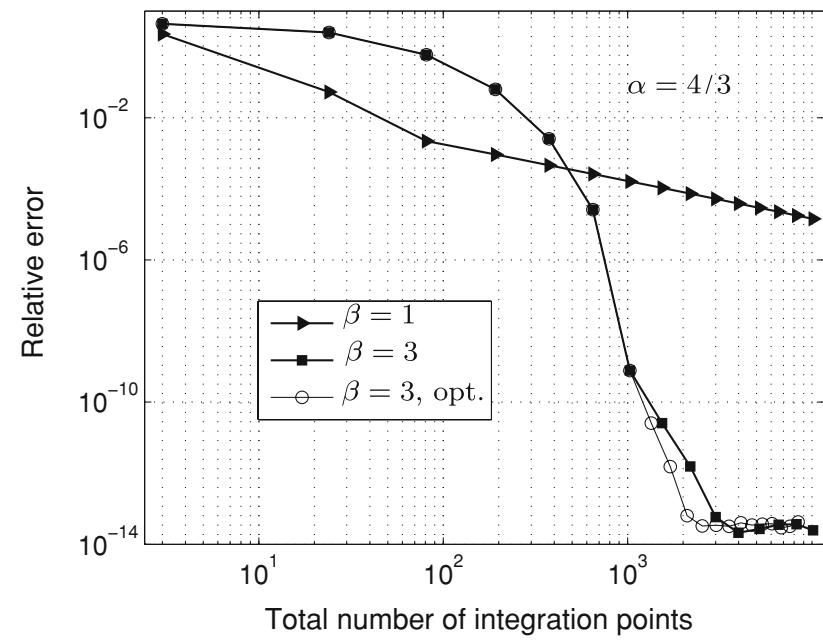

(b)

Fig. 5 Effect of optimization. (a) Unit square, $\alpha=1 / 2$ (compare to Fig. 2d); and (b) unit cube, $\alpha=4 / 3$ (compare to Fig. 4g)

$$
\begin{aligned}
& \int_{\Omega} \nabla w \cdot \nabla u d V=\int_{\Gamma_{N}} w g d S \quad \forall w \in \mathcal{U}, \\
& \mathcal{U}=\left\{w: w \in H^{1}(\Omega), w=0 \text { on } \Gamma_{D}\right\},
\end{aligned}
$$

where $H^{1}(\Omega)$ is the Sobolev space that consists of functions and their derivatives that are square integrable in $\Omega$. The PUFE approximation for the trial function $u$ is [9]:

$u^{h}(\mathbf{x})=\sum_{i \in I} N_{i}(\mathbf{x}) u_{i}+\sum_{j \in J} N_{j}^{\mathrm{PU}}(\mathbf{x}) \psi(\mathbf{x}) a_{j} \equiv \sum_{k \in K} \Phi_{k}(\mathbf{x}) d_{k}$,

where $N_{i}(\mathbf{x})$ are FE basis functions, $N_{j}^{\mathrm{PU}}(\mathbf{x})$ are the FE basis functions used to form the enriched basis function, $\psi(\mathbf{x})$ is the enrichment function, and $u_{i}$ and $a_{j}$ are nodal coefficients associated with the finite element and enriched bases, respectively. On substituting the above trial function and using $\Phi_{k}$

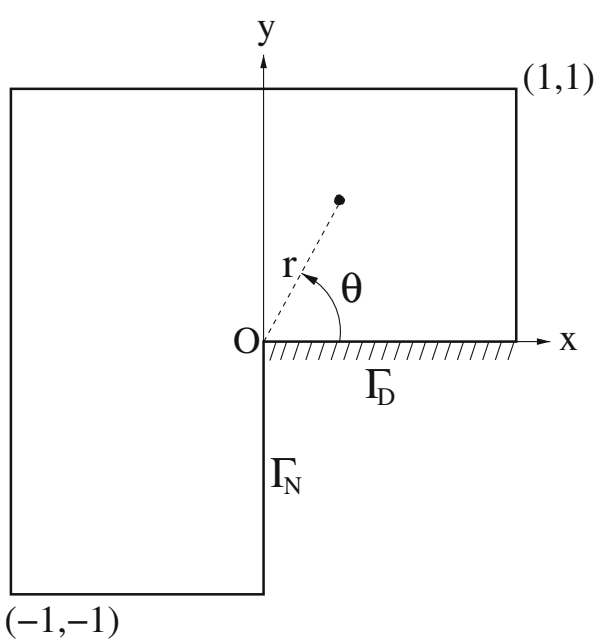

Fig. 6 Laplace problem on an $L$-shaped domain

as test functions in the weak form (4), we obtain the following discrete system of equations:

$$
\begin{aligned}
& \mathbf{K d}=\mathbf{f}, \quad \mathbf{d}=[\mathbf{u} \mathbf{a}]^{T}, \\
& \mathbf{K}_{i j}=\int_{\Omega} \nabla \Phi_{i} \cdot \nabla \Phi_{j} d V, \quad \mathbf{f}_{i}=\int_{\Gamma_{N}} \Phi_{i} g d S,
\end{aligned}
$$

where $\Phi_{i}=N_{i}$ for a classical degree of freedom and $\Phi_{i}=$ $N_{i}^{\mathrm{PU}} \psi$ for an enriched degree of freedom.

In this paper, we use linear ( $p=1, \mathrm{Q} 4)$, quadratic ( $p=2$, Q8) and cubic ( $p=3, \mathrm{Q} 12)$ serendipity finite elements, which are shown in Fig 7. The partition of unity enriched basis is always constructed as the product of the bilinear finite element basis function $\left(N_{i}^{\mathrm{PU}}=N_{i}^{\mathrm{Q} 4}\right)$ and the enrichment function. As in Ref. [12], we use $\psi(\mathbf{x})=r^{1 / 3} \sin (\theta / 3)$ as the enrichment function in the PUFE method. The DECUHR adaptive algorithm [57] (restricted to hyper-rectangular regions) was adopted by Strouboulis et al. [12] to compute the enriched contributions in the stiffness matrix, whereas the generalized Duffy transformation is used in this study.

In Fig. 8, a sample mesh of the domain with eight divisions along each coordinate direction is presented. First, we only enrich the node at the origin (one additional degree of freedom), which corresponds to an enrichment support radius $r_{e}=\epsilon(\epsilon$ is a small number). The support of the enriched basis function is the shaded region in Fig. 8. For FE computations, we use 4, 8, 16, 32, 64 and 128 number of divisions along each coordinate direction, and for PUFE computations, meshes with 4, 8, 16, and 32 divisions are chosen. Table 1 shows the number of degrees of freedom for the different meshes and element types. For FE stiffness matrix calculations and for elements that do not contain an enriched node in PUFE calculations, $4 \times 4$ tensor-product Gauss quadrature rule is used. For stiffness matrix calculation of the three elements containing the singularity (shaded elements in Fig. 8), 


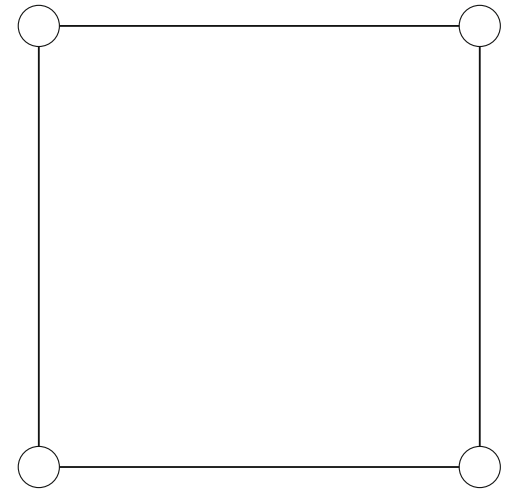

(a)

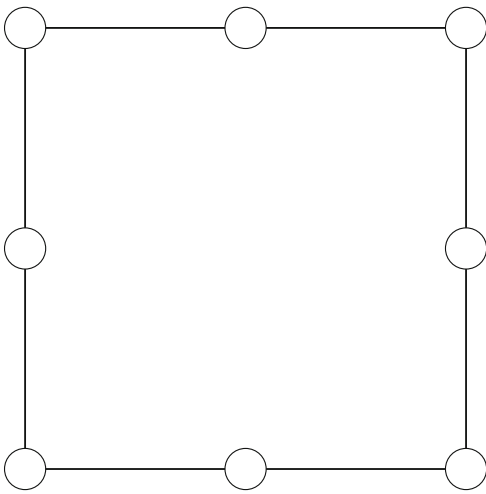

(b)

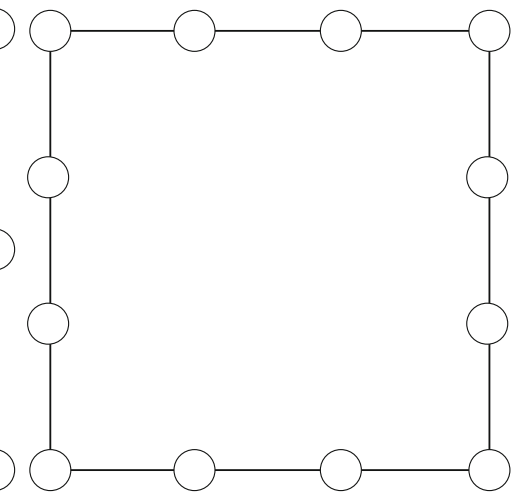

(c)

Fig. 7 Serendipity elements. (a) Linear (Q4, $p=1)$; (b) Quadratic (Q8, $p=2)$; and (c) Cubic (Q12, $p=3)$

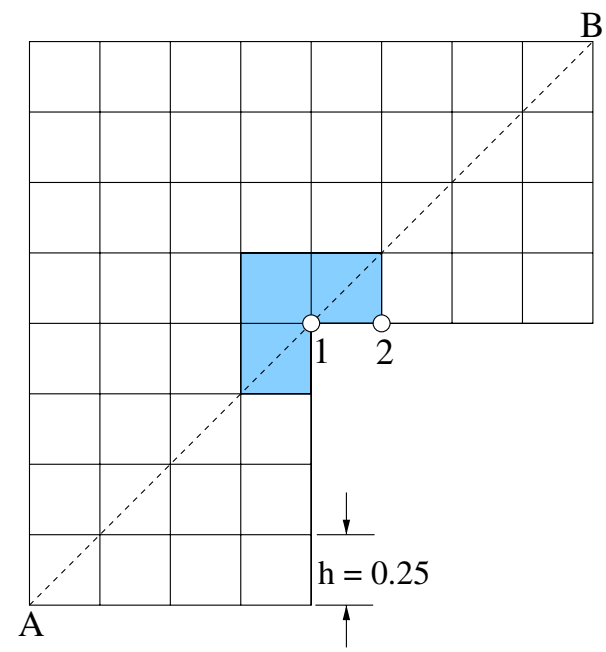

Fig. 8 Sample mesh $(8 \times 8$ mesh divisions $)$ for $L$-shaped domain

we use a $10 \times 10$ generalized Duffy quadrature rule (total of 600 evaluation points over the three elements) in the PUFE computations.

Figure 9a shows a plot of the solution along the diagonal $A B$ (see Fig. 8) for FE with different $p$ th order elements and Fig. $9 \mathrm{~b}$ and $\mathrm{c}$ show the results for PUFE. In the PUFE plots, the contributions due to the FE basis and the enriched basis are depicted separately. As seen in these figures, the
PUFE solution is proximal to the exact solution and is able to capture the sharp gradients of the solution in the vicinity of the singular point. As a measure of accuracy of the methods, convergence in the strain energy is studied. The relative error in the strain energy is defined as:

$E=\frac{a(u, u)-a\left(u^{h}, u^{h}\right)}{a(u, u)}, \quad a(u, u)=\int_{\Omega} \nabla u \cdot \nabla u d V$,

where $a(u, u) / 2$ is the exact strain energy. The exact strain energy for the problem under consideration is: $E_{\mathrm{ex}}=$ 0.423569003301483. Figure 9d shows the convergence for FE and PUFE with $h$ and $p$-refinements. For all FE computations, the relative error remains greater than $\mathrm{O}\left(10^{-2}\right)$, whereas even on coarse meshes the accuracy of PUFE (one extra degree of freedom in comparison to the corresponding FE problem) is superior and reaches relative errors of $10^{-4}$ on a cubic mesh with 32 divisions along each coordinate direction. Due to the singularity $(\lambda=1 / 3)$ at the corner, however, the theoretical asymptotic rate of convergence in strain energy for finite elements is $\min (2 p, 2 \lambda)=2 \lambda=2 / 3$ [58]. The rates for all the curves in Fig. $9 d$ for $p$ th order FE and PUFE are in agreement with theory. This sub-optimal convergence was also noted in previous studies where enrichment for crack problems $(\lambda=1 / 2)$ is used $[49,50]$. We return to a potential remedy for this issue later on.
Table 1 Number of degrees of freedom for meshes used in FE and PUFE computations

\begin{tabular}{|c|c|c|c|c|c|c|c|c|c|}
\hline \multirow[t]{2}{*}{ Divisions } & \multicolumn{3}{|l|}{ FE } & \multicolumn{3}{|c|}{$\operatorname{PUFE}\left(r_{e}=\epsilon\right)$} & \multicolumn{3}{|c|}{$\operatorname{PUFE}\left(r_{e}=0.5\right)$} \\
\hline & Q4 & Q8 & Q12 & Q4 & Q8 & Q12 & Q4 & Q8 & Q12 \\
\hline 4 & 21 & 53 & 85 & 22 & 54 & 86 & 26 & 58 & 90 \\
\hline 8 & 65 & 177 & 289 & 66 & 178 & 290 & 77 & 189 & 301 \\
\hline 16 & 225 & 641 & 1,057 & 226 & 642 & 1,058 & 266 & 682 & 1,098 \\
\hline 32 & 833 & 2,433 & 4,033 & 834 & 2,434 & 4,034 & 989 & 2,589 & 4,189 \\
\hline 64 & 3,201 & 9,473 & 15,745 & - & - & - & 3,815 & 10,087 & 16,359 \\
\hline 128 & 12,545 & 37,377 & 62,209 & - & - & - & - & - & - \\
\hline
\end{tabular}




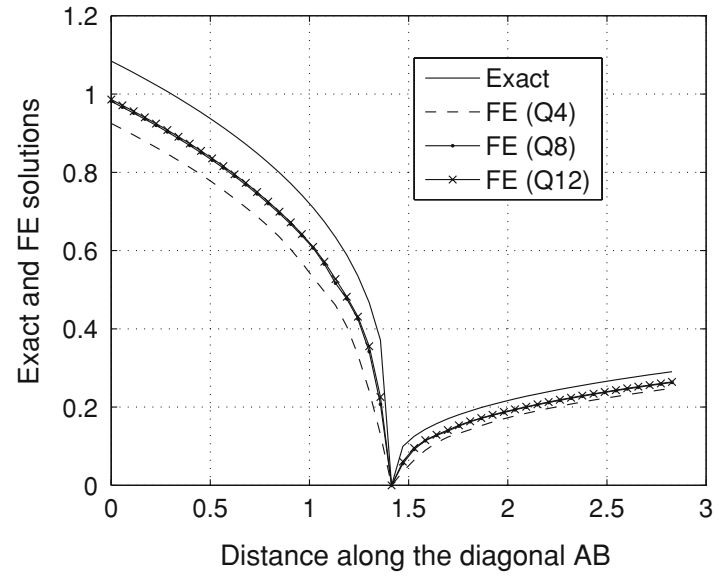

(a)

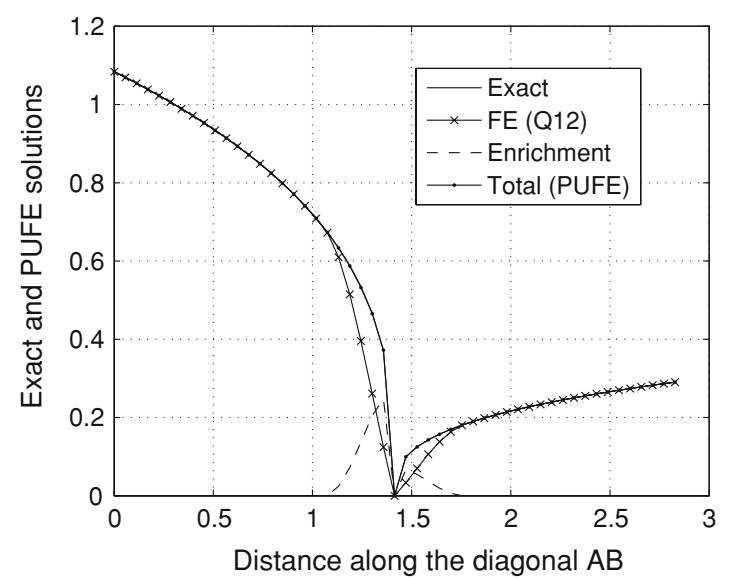

(c)

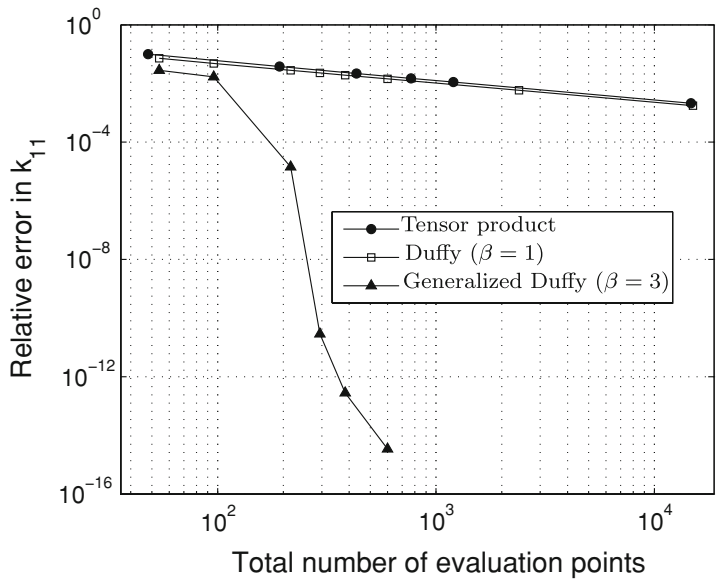

(e)

Fig. 9 Convergence study for FE and PUFE solutions. In the PUFE computations, only the vertex node is enriched $\left(r_{e}=\epsilon\right)$. (a) Solution along the diagonal $A B$ for $\mathrm{FE}$ with different element types; (b, c) Solution along the diagonal $A B$ for higher-order PUFE;

In Fig. 9e, the relative error in the computation of $k_{11}$ (the entry in the global stiffness matrix corresponding to the enriched degree of freedom assigned to the node at the origin;

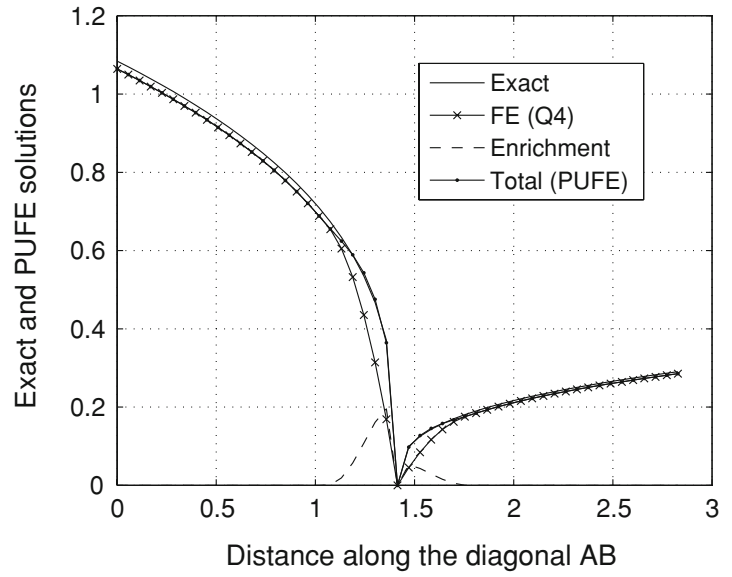

(b)

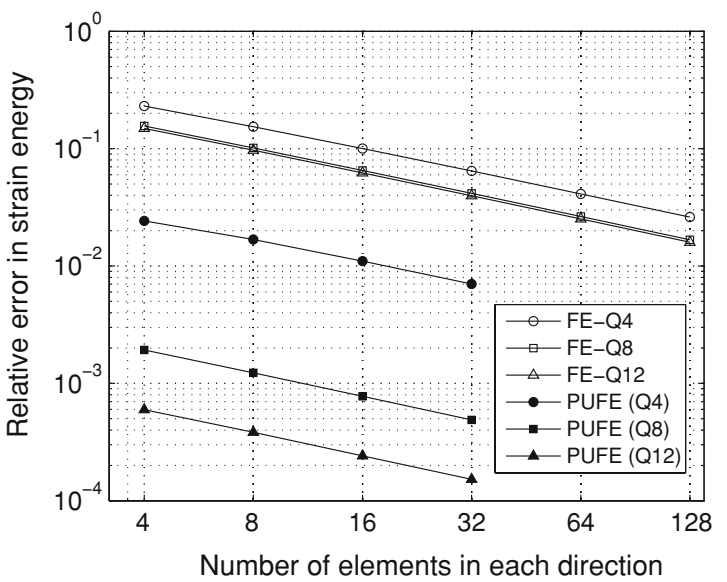

(d)

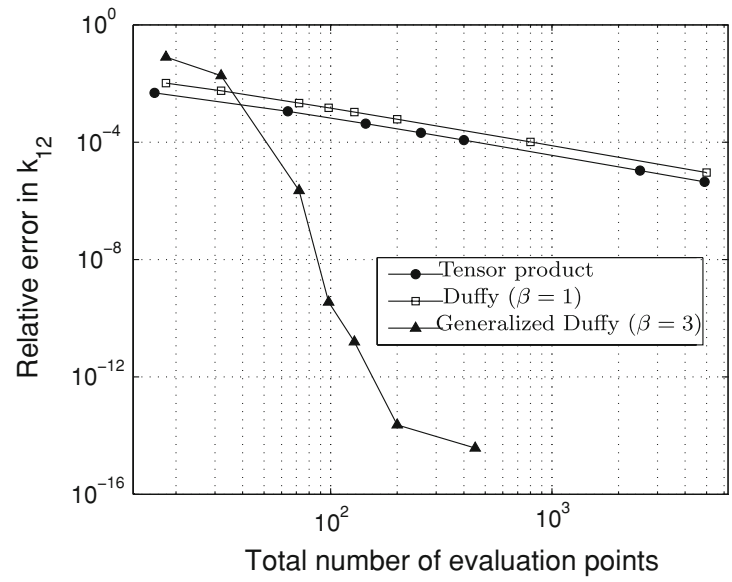

(f)

(d) Convergence of strain energy for FE and PUFE using the generalized Duffy transformation with $\beta=3$; and (e, f) Convergence of $k_{11}$ and $k_{12}$ for PUFE $(8 \times 8$ mesh divisions $)$ using different integration schemes

see Fig. 8) is plotted for a tensor-product Gauss rule, using Duffy transformation, and by the generalized Duffy transformation that is proposed in this paper. The expression for $k_{11}$ 


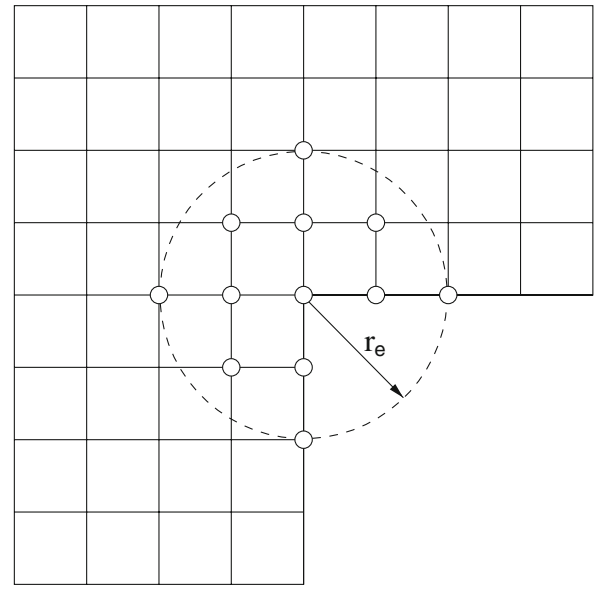

(a)

Fig. 10 Geometric enrichment for Laplace problem on an $L$-shaped domain. (a) Sample mesh, enriched nodes $\left(r_{e}=0.5\right)$ are shown by open circles; and (b) Convergence in strain energy for PUFE using

contains terms with $1 / r^{\alpha}$ singularities, where $\alpha=1 / 3,4 / 3$ :

$k_{11}=\int_{\Omega_{1}} \nabla\left(N_{1} \psi\right) \cdot \nabla\left(N_{1} \psi\right) d V \equiv \int_{\Omega_{1}} K(\mathbf{x}) d V$,

where $\psi=r^{1 / 3} \sin (\theta / 3), N_{1}$ is the bilinear finite element basis function associated with node 1 and $\Omega_{1}$ is its support (shaded region in Fig. 8). On letting $\mathbf{q}=-\sin (2 \theta / 3) \mathbf{i}+$ $\cos (2 \theta / 3) \mathbf{j}$ and using (3), we can write the integrand $K(\mathbf{x})$ as

$$
\begin{aligned}
K(\mathbf{x})= & r^{2 / 3} \sin ^{2}(\theta / 3)\left\|\nabla N_{1}\right\|^{2}+\frac{2 \sin (\theta / 3) N_{1} \nabla N_{1} \cdot \mathbf{q}}{3 r^{1 / 3}} \\
& +\frac{N_{1}^{2}}{9 r^{4 / 3}} .
\end{aligned}
$$

In Fig. 9f, the relative error in the computation of $k_{12}$ (corresponding to the enriched degree of freedom of node 1 and the classical degree of freedom of node 2 (see Fig. 8), is presented. The expression for $k_{12}$ contains a term with $r^{-2 / 3}$ singularity:

$k_{12}=\int_{\Omega_{2}} \nabla\left(N_{1} \psi\right) \cdot \nabla N_{2} d V \equiv \int_{\Omega_{2}} K(\mathbf{x}) d V$,

where $N_{i}(i=1,2)$ are bilinear finite element basis functions, $\Omega_{2}$ is the element that contains nodes 1 and 2 in its connectivity, and $K(\mathbf{x})$ in this case is given by

$K(\mathbf{x})=r^{1 / 3} \sin (\theta / 3) \nabla N_{1} \cdot \nabla N_{2}+\frac{N_{1} \nabla N_{2} \cdot \mathbf{q}}{3 r^{2 / 3}}$.

A benefit that accrues with the generalized Duffy transformation is that $\beta=3$ can be used to integrate all the terms in the above integrals. As can be discerned from Fig. 9e and $\mathrm{f}$, the generalized transformation integrates both $k_{11}$ and $k_{12}$ to almost machine precision with about 200 function evaluations per element ( $k_{11}$ is computed over three elements whereas $k_{12}$ is evaluated on one element). For computing $k_{11}$, 600 function evaluations $(10 \times 10$ over each triangle after the square elements are triangulated) are required, whereas the Duffy transformation $(\beta=1)$ minimally improves the tensor product rule, a consequence that bears out since the $1 / r^{4 / 3}$ singularity $(\alpha=4 / 3>1)$ is not removed by the Duffy transformation. It is noteworthy to point out that the generalized Duffy transformation outperforms the adaptive algorithm used in Ref. [12]. The relative error for $k_{11}$ in Ref. [12] is $10^{-5}$ for about 400 evaluation points; with the generalized Duffy transformation the same accuracy is attained with just over 200 evaluation points and more importantly, the error can be further reduced without significant addition in the number of evaluation points.

To address the issue of sub-optimality in the rate of convergence of PUFE with topological enrichment (see Fig. 9d), Laborde et al. [49] and Béchet et al. [50] suggested the notion of geometric (fixed-area) enrichment—all nodes that are inside a fixed region of the singularity are enriched. We choose an enrichment radius $r_{e}=0.5$ and all nodes within a distance of $r_{e}$ or less from the vertex are enriched (see Fig. 10a). The number of degrees of freedom in the PUFE computations are listed in Table 1. Figure 10b shows the convergence curves for the strain energy using linear, quadratic, and cubic PUFE solutions. Duffy transformation $(\beta=1)$ with a $16 \times 16$ tensor-product rule is compared to the generalized transformation $(\beta=3)$ with a $10 \times 10$ tensor-product rule. With the generalized Duffy quadrature, we obtain rates of convergence in strain energy of $1.85,3.92$ and 5.92 (rates are computed for PUFE solutions using 16, 32, and 64 divisions) for 
the linear, quadratic and cubic elements, respectively, which indicates that close to the optimal $2 p$ rate of convergence is recovered. Hence, the generalized Duffy quadrature is accurate and yields the correct rate of convergence, whereas the Duffy quadrature fails (non-monotonicity and incorrect rate of convergence). To ensure convergence, the quadrature error must be at least an order smaller than the PUFE approximation error, which is the case when the generalized Duffy transformation is used, but is not so when the Duffy transformation (see Fig. 9e, f) or a tensor-product Gauss rule is applied.

\section{Conclusions}

In this paper, we presented a generalization to the well-known Duffy transformation, which has been widely used for integration of kernels having $1 / r$ singularity. We introduced the following generalized Duffy transformation: $(u, v, w) \rightarrow$ $(x, y, z): x=u^{\beta}, y=u^{\beta} v, z=u^{\beta} w$, with $\beta$ as an additional parameter. The choice of $\beta$ was guided by the observation that the transformed kernel not have a fractional exponent. For instance, for $\alpha=\{1,1 / 2,1 / 3,2 / 3,4 / 3\}, \beta=$ $\{1,2,3,3,3\}$ was the optimal choice, respectively. All cases were tested in two and three dimensions, and the numerical results clearly demonstrated the superior accuracy and efficiency of the generalized transformation over the standard Duffy transformation. We also showed that the number of evaluation points can be further reduced by using the minimum number of Gauss points in $u$-direction (both in two- and three-dimensional applications) so that integration is carried out exactly with respect to $u$ and higher-order quadrature rules are used in the other directions.

The $L$-shaped domain with a corner singularity was considered, and higher-order FE and PUFE computations were performed. Once again, the merits of the generalized Duffy transformation were revealed in the computation of the enriched stiffness matrix entries. The convergence in strain energy of PUFE was studied: when only the vertex node was enriched, a convergence rate of 0.66 was realized, but when a fixed region of radius 0.5 was enriched, near-optimal $2 p$ rate of convergence was recovered. This was possible due to the highly accurate integration that the generalized Duffy transformation afforded; use of the Duffy transformation or a tensor-product Gauss rule led to inaccuracies since the quadrature error dominated the approximation error.

The generalized Duffy transformation can be implemented in boundary element and enriched finite element methods for the integration of singular functions without adding to the complexity of the programming and at the same time reducing the number of evaluation points with respect to the Duffy transformation. Furthermore, when there is a need for frequent integration of the singular kernel over the same domain, the generalized Duffy transformation can be combined with the node elimination algorithm presented in Mousavi et al. [59] to construct a very efficient quadrature rule with far fewer number of evaluation points. The term $1 / r^{\alpha}$ containing the singularity is used as the weight function in these quadrature rules similar to the quadratures presented by Haegemans [60]. Even though this study targeted integrands with vertex singularities in PUFE methods, the stringent demands on accuracy in non-singular PUFE applications such as acoustics [61] and Schrödinger and Poisson solutions in quantum mechanics [62] reinforces the need and importance of developing accurate and efficient quadrature rules for enriched finite element methods.

\section{Appendix A}

Generalized Duffy transformation in two dimensions The mapping $(u, v) \rightarrow(x, y): x=u^{\beta}, y=u^{\beta} v$ transforms the integral from the standard triangle (Fig. 1a) to the unit square (Fig. 1b):

$$
\begin{aligned}
\mathcal{I} & =\int_{0}^{1} d x \int_{0}^{x} d y \frac{f(x, y)}{\left(x^{2}+y^{2}\right)^{\alpha / 2}} \\
& =\int_{0}^{1} \int_{0}^{1} \frac{f\left(u^{\beta}, u^{\beta} v^{\gamma}\right)}{\left[u^{2 \beta}\left(1+v^{2 \gamma}\right)\right]^{\alpha / 2}} \mathcal{J} d u d v,
\end{aligned}
$$

where $\mathcal{J}$ is defined as

$\mathcal{J}=\left(\beta u^{\beta-1}\right)\left(\gamma u^{\beta} v^{\gamma-1}\right)=\beta \gamma u^{2 \beta-1} v^{\gamma-1}$.

On setting $\gamma=1$, we obtain

$\mathcal{I}=\int_{0}^{1} \int_{0}^{1} \frac{f\left(u^{\beta}, u^{\beta} v\right)}{\left(1+v^{2}\right)^{\alpha / 2}} \beta u^{2 \beta-1-\alpha \beta} d u d v$.

Any arbitrary triangle with a vertex singularity is first translated so that the singularity is moved to the origin and then an affine map to the standard triangle is used: $x=a X+b Y$ and $y=c X+d Y$, where $(X, Y)$ is the physical coordinate system containing the arbitrary triangle and $(x, y)$ is the plane of the standard triangle. Equivalently, given an arbitrary triangle $R$ having one vertex at the origin, there exists an affine transformation $\mathbf{A}$ that takes the standard triangle to $R$ [21]. The transformation $\mathbf{A}$ can be used to map the points of a rule over the standard triangle to $R$.

\section{Appendix B}

MATLAB code for construction of generalized Duffy quadrature in two dimensions 
11. Moës N, Dolbow J, Belytschko T (1999) A finite element method for crack growth without remeshing. Int J Numer Methods Eng 46(1):131-150

12. Strouboulis T, Babuška I, Copps K (2000) The design and analysis of the generalized finite element method. Comput Methods Appl Mech Eng 181(1-3):43-69

13. Hansbo A, Hansbo P (2004) A finite element method for the simulation of strong and weak discontinuities in solid mechanics. Comput Methods Appl Mech Eng 193(33-35):3523-3540

14. Huang R, Prèvost J-H, Huang ZY, Suo Z (2003) Channel-cracking of thin films with the extended finite element method. Eng Fract Mech 70:2513-2526

15. Giner E, Sukumar N, Fuenmayor FJ, Vercher A (2008) Singularity enrichment for complete sliding contact using the partition of unity finite element method. Int J Numer Methods Eng 76(9):14021418

16. Elguedj T, Gravouil A, Combescure A (2006) Appropriate extended functions for X-FEM simulation of plastic fracture mechanics. Comput Methods Appl Mech Eng 195:501-515

17. Bunger AP, Detournay E (2008) Experimental validation of the tip asymptotics for a fluid-driven crack. J Mech Phys Solids 56(11):3101-3115

18. Lecampion B (2009) An extended finite element method for hydraulic fracture problems. Commun Numer Methods Eng 25:121-133

19. Waisman H, Belytschko T (2008) Parametric enrichment adaptivity by the extended finite element method. Int J Numer Methods Eng 73:1671-1692

20. Lyness JN (1976) Applications of extrapolation techniques to multidimensional quadrature of some integrand functions with a singularity. J Comput Phys 20:346-364

21. Lyness JN (1992) On handling singularities in finite elements. In: Espelid TO, Genz A, (eds) Numerical integration, recent developments, software and applications, NATO ASI Series C: Mathematical and Physical Sciences, vol 357. Kluwer Academic Publishers, Dordrecht, pp 219-233

22. Espelid TO (1994) On integrating vertex singularities using extrapolation. BIT Numer Math 34:62-79

23. de Doncker E, Shimizu Y, Fujimoto J, Yuasa F (2004) Computation of loop integrals using extrapolation. Comput Phys Commun 159:145-156

24. Schwab C (1994) Variable order composite quadrature of singular and nearly singular integrals. Computing 53(2):173-194

25. Genz A, Cools R (1997) An adaptive numerical cubature algorithm for simplices. Technical Report TW 273, Department of Computer Science, K. U. Leuven, Belgium

26. Klees R (1996) Numerical calculation of weakly singular surface integrals. J Geod 70(11):781-797

27. Takahasi H, Mori M (1973) Quadrature formulas obtained by variable transformation. Numer Math 21(3):206-219

28. Duffy MG (1982) Quadrature over a pyramid or cube of integrands with a singularity at a vertex. SIAM J Numer Anal 19(6):12601262

29. Lean MH, Wexler A (1985) Accurate numerical integration of singular boundary element kernels over boundaries with curvature. Int J Numer Methods Eng 21:211-228

30. Aliabadi MH, Hall WS (1987) Analytical removal of singularities and one-dimensional integration of three-dimensional boundary element method kernels. Eng Anal 4:21-24

31. Schwab C, Wendland WL (1992) On numerical cubatures of singular surface integrals in boundary element methods. Numer Math 62(3):343-369

32. Nagarajan A, Mukherjee S (1993) A mapping method for numerical evaluation of two-dimensional integrals with $1 / r$ singularity. Comput Mech 12:19-26
33. Khayat MA, Wilton DR (2005) Numerical evaluation of singular and near-singular potential integrals. IEEE Trans Antennas Propag 53(10):3180-3190

34. Monegato G, Scuderi L (1999) Numerical integration of functions with boundary singularities. J Comput Appl Math 112(1-2):201214

35. Fairweather G, Rizzo FJ, Shippy DJ (1979) Computation of double integrals in the boundary integral equation method. In: Vichnevetsky R, Stepleman RS, (eds) Advances in computer methods for partial differential equations-III. IMACS Publ, Brussels, pp 331-334

36. Tracey DM (1971) Finite elements for determination of crack tip elastic stress intensity factors. Eng Fract Mech 3:255-265

37. Tracey DM, Cook TS (1977) Analysis of power type singularities using finite elemetns. Int J Numer Methods Eng 11:1225-1233

38. Stern M, Becker EB (1978) A conforming crack tip element with quadratic variation in the singular fields. Int J Numer Methods Eng $12: 279-288$

39. Ying L (1982) Some special interpolation formulas for triangular and quadrilateral elements. Int J Numer Methods Eng 18:959-966

40. Solecki JS, Swedlow JL (1984) On quadrature and singular finiteelements. Int J Numer Methods Eng 20:395-408

41. Varnhorn W (1989) Efficient quadrature for a boundary element method to compute three-dimensional Stokes flow. Int J Numer Methods Fluids 9(2):185-191

42. Harris PJ (1992) A boundary element method for the Helmholtz equation using finite part integration. Comput Methods Appl Mech Eng 95:331-342

43. Ling F, Liu J, Jin $\mathbf{J}$ (2002) Efficient electromagnetic modeling of three-dimensional multilayer microstrip antennas and circuits. IEEE Trans Microwave Theory Tech 50(6):1628-1635

44. Jørgensen E, Volakis JL, Meincke P, Breinbjerg O (2004) Higher order hierarchical Legendre basis functions for electromagnetic modeling. IEEE Trans Antennas Propag 52(11):2985-2995

45. Yu H, Bandrauk AD, Sonnad V (1994) Three-dimensional finite element method for electronic properties of small polyatomic molecules: $\mathrm{H}_{2}^{+}, \mathrm{H}_{2}, \mathrm{H}_{3}^{2+}$ and $\mathrm{H}_{3}^{+}$. Chem Phys Lett 222(4):387-393

46. Yu H, Bandrauk AD (1995) Three-dimensional Cartesian finite element method for the time dependent Schrödinger equation of molecules in laser fields. J Chem Phys 102(3):1257-1265

47. Batcho PF (2000) Computational method for general multicenter electronic structure calculations. Phys Rev E 61(6):7169-7183

48. Havu P, Havu V, Puska MJ, Nieminen RM (2004) Nonequilibrium electron transport in two-dimensional nanostructures modeled using Green's functions and the finite-element method. Phys Rev B 69(11):115325

49. Laborde P, Pommier J, Renard Y, Salaun M (2005) High-order extended finite element method for cracked domains. Int J Numer Methods Eng 64:354-381

50. Béchet E, Minnebo H, Moës N, Burgardt B (2005) Improved implementation and robustness study of the X-FEM for stress analysis around cracks. Int J Numer Methods Eng 64(8):1033-1056

51. Xiao QZ, Karihaloo BL (2006) Improving the accuracy of XFEM crack tip fields using higher order quadrature and statically admissible stress recovery. Int J Numer Methods Eng 66(9):1378-1410

52. Ventura G, Gracie R, Belytschko T (2009) Fast integration and weight function blending in the extended finite element method. Int J Numer Methods Eng 77(1):1-29

53. Park K, Pereira JP, Duarte CA, Paulino GH (2009) Integration of singular enrichment functions in the generalized/extended finite element method for three-dimensional problems. Int $\mathrm{J}$ Numer Methods Eng 78(10):1220-1257

54. Sukumar N, Kumosa M (1992) Application of the finite element iterative method to cracks and sharp notches in orthotropic media. Int J Fract 58(2):177-192 
55. Yosibash Z, Schiff B (1997) Superelements for the finite element solution of two-dimensional elliptic problems with boundary singularities. Finite Elem Anal Des 26(4):315-335

56. Liu X, Lee CK, Fan SC (2002) On using enriched cover function in the Partition-of-unity method for singular boundary-value problems. Comput Mech 29:212-225

57. Espelid TO, Genz A (1994) DECUHR: an algorithm for automatic integration of singular functions over a hyperrectangular region. Numer Algorithms 8:201-220

58. Strang G, Fix G (1973) An analysis of the finite element method. Prentice-Hall, Englewood Cliffs

59. Mousavi SE, Xiao H, Sukumar N (2009) Generalized Gaussian quadrature rules on arbitrary polygons. Int J Numer Methods Eng. doi:10.1002/nme.2759
60. Haegemans A (1993) Cubature formulas for triangles and squares with a $1 / r$ singularity. Technical Report TW 192, Department of Computer Science, K. U. Leuven, Belgium

61. Bettess P, Shirron J, Laghrouche O, Peseux B, Sugimoto R, Trevelyan J (2003) A numerical integration scheme for special finite elements for the Helmholtz equation. Int J Numer Methods Eng 56(4):531-552

62. Sukumar N, Pask JE (2009) Classical and enriched finite element formulations for Bloch-periodic boundary conditions. Int J Numer Methods Eng 77(8):1121-1138 